\title{
La Perspectiva Psicoanalítica Relacional en la Terapia de Pareja ${ }^{1}$
}

\author{
PHILIP A. RINGSTROM², PH.D., PSY.D. ${ }^{\mathrm{a}}$ \\ Los Ángeles (U.S.A.)
}

\begin{abstract}
Este trabajo intenta dar una respuesta a la pregunta ¿cómo un psicoanalista relacional puede conceptualizar la psicoterapia de pareja?. La respuesta aborda tres temas críticos que son relevantes para el psicoanálisis relacional en general, pero especialmente para el modelo de la terapia relacional de pareja: 1) la actualización del self comprometido a una relación de largo plazo, seguido por 2) la necesidad de reconocimiento mutuo versus la negación mutua (para que puedan tener lugar las actualizaciones del self); y por último, 3) algo inevitable que ocurre en las relaciones a largo plazo, algo que yo denomino: relación que tiene una mente propia. Tras ello se comenta la propuesta de los seis pasos que desarrolla mi modelo de terapia de pareja.
\end{abstract}

Palabras clave: Psicoanálisis Relacional, Psicoterapia de Pareja

This paper try to give answer to que question: how a relational psychoanalyst might conceptualize couple's psychotherapy?. The answer faces three critical themes: first, self-actualization in a long term committed relationship, followed by the second theme of the necessity of mutual recognition versus mutual negation (for self-actualization to be able to occur). Finally, came a third theme regarding something quite ineffable that occurs in long term relationships, which I refer to as the relationship having a mind of its own. Later, the six steps proposal of my Couple's Therapy model are discussed.

Key Words: Relational Psychoanalysis, Couple's Psychotherapy

English Title: Relational Psychoanalytic Perspective on Couples Psychotherapy

\section{Cita bibliográfica / Reference citation:}

Ringstrom, P.A. (2018). La perspectiva psicoanalítica relacional en la Terapia de Pareja. Clínica e Investigación Relacional, 12 (1): 60-77. [ISSN 1988-2939] [Recuperado de www.ceir.info] DOI:

10.21110/19882939.2018.120105

${ }^{1}$ Presentado en las $V$ Jornadas de Psicoanálisis Relacional, Organizadas por el Instituto de Psicoterapia Relacional (IPR), La Granja de San Ildefonso, Segovia, España, 23 de Febrero de 2018. Traducción castellana de N. Monserrat Gómez García, revisado por María Hernández Gázquez y Alejandro Ávila.

${ }^{2}$ Analista Didacta del Instituto de Psicoanálisis Contemporáneo; Miembro fundador del Board de la Asociación Internacional de Psicoanálisis y Psicoterapia Relacional (IARPP); Miembro del Consejo Internacional de Psicólogos del Self; Miembro del Consejo Editorial de Psychoanalytic Dialogues, The International Journal of Psychoanalytic Self Psychologists and Psychoanalytic Perspectives. Práctica Privada en Encino, California. 15527 Valley Vista Blvd. - Encino, CA 91436 - OFC: 818 906-8408 - FAX: 818 906-3269. 
Mucho de lo que presentaré el día de hoy fue extraído de un artículo que será publicado el próximo año en la revista americana Psychoanalytic Inquiry. La edición de esta revista, en donde se publicará el artículo, ha sido organizada por la editora Heather Maclntosh. Ella invitó a varios autores de diferentes perspectivas (relacional, relaciones objetales, clásico, psicología del self, y teoría de los sistemas intersubjetivos) para discutir sobre cómo nuestra teoría influye en nuestra aproximación a la terapia psicoanalítica de pareja. En mi caso, Heather me pidió que escribiese sobre cómo un psicoanalista relacional (las cursivas son del autor) podría conceptualizar la psicoterapia de pareja.

Al aceptar su petición, pensé de inmediato que la mejor manera para ilustrar el psicoanálisis relacional en detalle es a través de la lente del trabajo con parejas. De hecho, una de las primeras ideas que siempre introduzco al presentar mi modelo de tratamiento con parejas es que demuestra inmediatamente la transferencia de una forma que, a menudo, es más difícil de hacer en una terapia psicoanalítica individual -especialmente en pacientes poco familiarizados y, por lo tanto, más intimidados que intrigados con el psicoanálisis.

Como ejemplo, ofrezco la diferencia entre la reacción de un paciente en terapia individual por mi retraso de cinco minutos en nuestra sesión con el objetivo de explorar la potencial reacción transferencial. "¿Cuáles son tus fantasías del porqué he llegado tarde hoy?" Para el paciente promedio, familiarizado con la indagación psicoanalítica, esta pregunta podría parecerle un poco "loca y rara" para él, por lo menos inicialmente. Sin embargo, si cambiamos esta ilustración a una terapia de pareja y su esposa llega cinco minutos tarde, podríamos ver algo totalmente diferente. Para seguir ilustrando, imaginemos que, a diferencia de su "desconcertada" e incómoda reacción a mi indagación sobre sus "fantasías" acerca de mi impuntualidad, en el tratamiento con parejas él se torna furioso al instante con su mujer, de una manera relativamente cruda y sin restricciones.

Lo que organiza inmediatamente el campo de esta sesión de pareja es lo que el "retraso" de su esposa significa para él. Ese significado es muy probable que sea más compatible con su sensibilidad que uno planteado por su terapeuta en psicoterapia individual. Por su reacción en la sesión de pareja, emerge una investigación sobre la experiencia histórica que tiene de la impuntualidad de los demás y lo que significa esto para él. Otra vez, esto se trata menos de sus "fantasías" de lo que trama su "extraño" terapeuta individual que sobre su cruda experiencia en la sesión de terapia de pareja.

Jugando con esta ilustración aún más, imaginemos que nos enteramos de que, cuando era niño, su madre siempre pasaba tarde a recogerlo del colegio. Sentado solo, mientras el sol se ponía, con poca predicción de su tiempo de llegada, comprensiblemente 
lo llenó de temor, miedo y aprensión. Eventualmente, sin embargo, en un intento fallido de afrontarlo, asumió una especie de depresión disociada, aunque no surge finalmente en ira hacia su esposa.

Esto, espero que podamos estar de acuerdo, ofrece una evidencia intachable de su palpable reacción transferencial hacia el retraso de su esposa. Mientras tanto, ya que esto es terapia de pareja, ahora podemos indagar de qué va el crónico retraso de ella. Quizá en su narrativa, aprendemos que representa un tipo de transferencia oposicionista hacia su padre por haberla reprendido constantemente en su juventud. En resumen, estamos inmediatamente en el dominio de las transferencias de ambos compañeros en conjunto con cómo esto se está volviendo sistemáticamente orquestado en un círculo vicioso en su relación, por ejemplo, su impuntualidad activa (dispara) su ira y su ira exacerba más el que ella, en oposición, llegue tarde.

Retomando el tema contemporáneo de lo Relacional, sabemos que el problema principal que enfrenta el Psicoanálisis del siglo 21 es que, del nacimiento a la muerte, cada aspecto de la psicología humana esta profunda e inextricablemente vinculada a las relaciones. Esto incluye lo que sucede en el plano interaccional de la realidad. Pero aún así, involucra lo que está sucediendo en nuestras fantasías, junto con cómo representamos nuestro sentido del "self" en relación con los otros, así como ellos con nosotros.

En el caso del marido y la mujer, el problema de la impuntualidad tiene gran impacto porque, cuando se manifiesta en su relación, está activas un cúmulo de imágenes negativas y de fantasías de sus respectivos pasados. Entonces, mientras que todas las relaciones operan en la factibilidad del aquí y ahora, en terapia de pareja, es más sobre las "fantasías" que se agitan como resultado de su interacción. Esto crea un enfoque de tratamiento similar al que presentaré el día de hoy, por excelencia uno que gire en torno a la teoría y práctica psicoanalítica.

Quizá mejor concebida como una "teoría de campo"bl (Stern, 2015; Ringstrom, en prensac) el psicoanálisis relacional presenta una variedad de conceptos relevantes para lo que sucede cuando se mira a través del lente de ideas como: teoría del reconocimiento, intersubjetividad, procesos de disociación, múltiples estados del self, enactments, así como con lo que pasa cuando el enfoque observacional del terapeuta cambia una y otra vez de observador-intérprete a participante-observador y a participante-participante. Esta breve lista conceptual se vuelve el medio por el cual todas las ideas principales del psicoanálisis evolucionan de ser predominantemente psicología individual, la llamada "psicología de una persona", a una "teoría de campo" concebida ahora en términos de una "psicología multipersonal." expresa. Este material es para uso científico y profesional exclusivamente y puede contener información clínica sensible. Los editores no se responsabilizan de los contenidos de los autores. Dirigir las consultas sobre derechos y autorizaciones a ceir@psicoterapiarelacional.es 
Fue desde este punto de vista relacional que desarrollé mi modelo de terapia de pareja que se puede encontrar en mi libro A Relational Psychoanalytic Approach to Couples Psychotherapy (2014). Este libro fue la evolución de mi pensamiento y práctica por más de cuatro décadas. Esta estadía comenzó con mi insatisfacción con el psicoanálisis cuando me formaba en la universidad a principios de 1970, lo cual me lanzó hacia las ideas de la teoría familiar sistémica. Por una década y media intenté entender cuál de las teorías sistémicas familiares me quedaba mejor ya que existía un gran desacuerdo entre ellas. Por ejemplo, durante ese periodo, la teoría familiar sistémica se esparcía a lo largo de un continuo de, por un lado, aquellas teorías tan casadas con los "sistemas" que el mero constructo de "self" era aborrecible. Por otra parte, había teorías que se mantenían enganchadas con el concepto del self. Estas últimas teorías estaban casadas con la importancia de luchar con lo que significa ser uno mismo en un sistema. Uno que encarna las influencias que están por siempre cambiando, retando e inclusive, en circunstancias psicopatológicas, amenazando la existencia del propio sentido del self.

En mi mente, los más estridentes pensadores de sistemas fueron muy lejos al apropiarse del sentido del self como algo inusual o un tanto "delirante". Especialmente cuando es comparado con la inexorable maquinación del sistema contextual. Su énfasis socavó para mí lo que significaba ser un individuo en un sistema. Esto es, para que uno se sienta emotivo, el crear y perseguir los sueños de uno mientras aprende a enfrentar las vicisitudes de la propia existencia y ontología. Y finalmente, para poder enfrentar las inherentes confusiones de enfrentarse a la propia mortalidad.

En breve, no pude abandonar el sentido de lo que ser uno mismo involucra, no importa que tan confuso se haya vuelto (lo confuso que se haya vuelto) frente al poder de una perspectiva sistemática. Para mí, un sistema sin un concepto de self era simplemente inaceptable. Aún, sin importar lo que la experiencia del self signifique para uno, tampoco puede realizarse del todo a menos que encuentre su lugar en un sistema, especialmente en relación con el cual se desarrolló. Por lo tanto, un self sin un sentido de cómo está colocado en un sistema era algo simplemente inaceptable.

Esto me llevó a mi contemplación de tres temas críticos que son relevantes para el psicoanálisis relacional en general, pero especialmente para el modelo de terapia de pareja que estaba concibiendo. Estos temas toman primero la actualización del self comprometido a una relación de largo plazo, seguido por el segundo tema de la necesidad de reconocimiento mutuo versus la negación mutua (para que puedan tener lugar las actualizaciones del self). Por último, viene el tercer tema que trata sobre algo inevitable que 
ocurre en las relaciones a largo plazo, algo que yo llamo la relación que tiene una mente propia.

La organización de estos tres temas me permitió crear un viable acercamiento psicoanalítico relacional a la psicoterapia de pareja. Uno que verdaderamente se ajustaba a mi sensibilidad relacional. Lo que sigue es una breve descripción de lo que representan cada uno de estos temas, después de los cuales, surgirán los conceptos clave que emergen de ellos en la forma de un enfoque psicoanalítico sistemático y relacional de Seis Pasos para la psicoterapia de pareja.

El primer tema, la "actualización de experiencias del self en una relación íntima", traza sus orígenes a los comienzos de la Revolución Industrial hace 200 años. Debido a la explosión en tecnología y manufactura en los Estados Unidos y Europa, las mayores migraciones poblacionales comenzaron trasladándose de la vida agraria del campo a la vida metropolitana en donde los empleos en manufactura y todas las industrias subyacentes (por ejemplo, finanzas y banca) iban en explosivo incremento. Antes de la Revolución Industrial, la proporción de vida agraria en comparación con la metropolitana era de 90\% en el campo y $10 \%$ en la ciudad. Pero para cambio del siglo 20 aquellas proporciones en muchos lugares se revirtieron literalmente. Esto creó un giro titánico en la cultura familiar.

Con la masiva disrupción de la comunidad agraria basada en una vida familiar multigeneracional, el matrimonio cambió de ser un acuerdo social entre familias a ser más una elección personal. Eso significaba que la "selección de pareja", que antes era enteramente un arreglo social entre familias, se volvió una cuestión de elección entre parejas: Con este último giro, la elección de pareja de pronto se volvió fuertemente influenciada por el deseo. La Era Romántica en el arte y la literatura también habían comenzado y, por supuesto, tan pronto como la elección de pareja se volvía una cuestión de deseo, el mundo de la mente inconsciente se volvía un factor importante en la toma de esa elección. No nos olvidemos que la palabra Latina para deseo es ¡libido! No debería de ser sorprendente el que el psicoanálisis llegará a ser precisamente en el cambio del siglo 19 al 20.

La "actualización de la experiencia del self dentro de una relación íntima" se refiere a cómo los compañeros se atraen mutuamente fuera de un anhelo (deseo) por completar algo incompleto en su desarrollo. Esto se manifiesta en la convicción inconsciente de que: "Con este compañero, espero ser capaz de actualizar lo que hasta ahora no he podido. Sin embargo, con este compañero, también el temor a volver a visitar algún aspecto de mi pasado. De hecho, algo que desearía poder superar." Por supuesto, las ideas que son centrales para este primer tema surgen del trabajo de Stephen Mitchell (1993) sobre "esperanza y temor" junto con muchos otros en el canon relacional. 
Otra idea clave en este primer tema es que mucho de lo que observamos como actualización del self debe ocurrir en relación con los otros. Con esto me refiero a que nuestras más óptimas y satisfactorias experiencias de actualización del self usualmente requieren el reconocimiento de ellas por nuestro compañero. Sin embargo, conceptos críticos a la actualización de experiencia del self incluyen una experiencia de agencia y autoría de la propia vida, por las cuales ambas necesitan reconocimiento (Benjamin, 1988) para sentirse plenamente realizadas. En consecuencia, lo que se espera puede ponerlo en peligro a través de un reconocimiento erróneo. Esto vuelve la esperanza por reconstituir el propio crecimiento o el reparar lo que se ha roto como el único contrapeso con el temor de que las esperanzas de uno sean aplastadas que lleva a "volver a herir", sino es que posiblemente a la retraumatización.

El segundo tema del reconocimiento mutuo versus negación mutua, esta profundamente en deuda con el concepto de Jessica Benjamin de intersubjetividad en el cual el reconocimiento mutuo representa un paso evolutivo en la teorización psicoanalítica. La clave para el proceso de reparar rupturas es que las diadas maritales necesitan desarrollar la capacidad intersubjetiva para moverse más allá de la relación sujeto-a-objeto y la relación sujeto-a-sujeto. Esto es, de un ser humano (el "objeto") sirviendo o quizá frustrando las necesidades del otro (el "sujeto") ya sea por hecho o fantasía. La intersubjetividad Relacional involucra la cultivación de la curiosidad naciente de cada compañero acerca de su propia subjetividad y la de su pareja, de ahí la relación sujeto-asujeto.

Sin esto, las relaciones maritales potencialmente se transforman en patrones de "dominio y sumisión", lo cual Benjamin refiere como "complementariedad dividida". El Reconocimiento mutuo, sin embargo, no aspira al "gemeleo" del perpetuo mito de "sintonía mutua". En efecto, el reconocimiento mutuo no tiene mucho sentido visto independientemente de su posición dialéctica con "negación mutua". De esta manera, estamos intrínsecamente atascados en la fundamental "paradoja de reconocimiento". Incorporado en él está, la tensión dialéctica perpetua entre la afirmación del self versus el reconocimiento del otro. El primero implica el aspecto central del self de la actualización del self (a veces siendo "ruda", es decir, sin enfocar el cuidado y preocupación del otro por las propias necesidades y deseos) yuxtapuestas con la necesidad del otro por el reconocimiento de uno (incluyendo el ser rudo). Ser "rudo" o "despiadado" se complica fácilmente en la dialéctica del interés propio versus la afiliación.

Esto después lleva al tercer tema que es, la relación teniendo una mente propia. Con el gran impacto de la teoría general de sistemas en 1970, el psicoanálisis pasó por un 
masivo cambio paradigmático. Ya no había lugar para la linealidad del pensamiento de "causa y efecto". La premisa fundamental de la teoría de sistemas es que "todo influencia todo". Por lo tanto, las ideas de causa y efecto tales como "impulsos", ya no tenían dominio sobre el psicoanálisis, el cual fue gradualmente cambiando de ver los "impulsos" como algo fundamentalmente causal, a verlos como algo más parecido los sistemas motivacionales que surgen o son inhibidos en contextos relacionales (Lichtenberg, Lachmann y Fosshage, 1992 y 1996). Esto cimentó nuestra comprensión de que todas las relaciones humanas son circularmente causales, queriendo decir inextricablemente que son mutuamente influenciables y mutuamente reguladas.

Para 1990, la teoría general de sistemas se actualizaba con la teoría de sistemas dinámicos no-lineales, comúnmente conocida como teoría de la complejidad. Sus ideas principales de la teoría de la complejidad que establecen algunas bases al tercer tema de la relación con una mente propia, una base sobre la cual muchas ideas psicoanalíticas se construyen. En breve, aquí can (caben) algunas ideas de la teoría de la complejidad. Por ejemplo, "la sensible dependencia en condiciones iniciales" (el llamado "efecto mariposa"d) en el cual es imposible determinar cuál fue la condición inicial que estableció el sistema sobre examinación en movimiento. Extrapolando este principio en mi tema, a pesar de la tendencia de las parejas para hacerlo, es mucho más infructuoso el asignar la culpa en una relación porque lo que haya pasado inicialmente, podría haber dado origen a la atribución de la culpa, es fundamentalmente indeterminable. Esto se vuelve importante para ayudar a las parejas en deshacer su tendencia binaria de crear un sistema de culpabilizador y culpabilizado, que será retomado en mayor detalle en breve.

Los sistemas complejos pueden también, en formas altamente impredecibles, cambiar completamente en "puntos de criticabilidad", o llamados "puntos de inflexión". Esto es, el proverbio "la pajilla que rompió el lomo del camello" (esta es la expresión equivalente en castellano a la gota que colmó el vaso que en inglés no existe). En estos y otras formas, los sistemas de la complejidad prueban ser dinámicos, fluidos y altamente impredecibles. También son reactivos a "perturbaciones" que involucran la ocurrencia de cosas que son fuertemente disruptivas a las operaciones normativas de cualquier sistema.

Al desarrollar el tercer tema, la relación teniendo una mente propia, muchas ideas psicoanalíticas importantes pasaron por mi mente que fácilmente se conectaban con la teoría de la complejidad. La primera de ellas es el concepto de Thomas Ogden (1994) del tercero analítico - su modelo de intersubjetividad - en donde los pensamientos de cada compañero inextricablemente se influencian la una a la otra uno a otro en un proceso tal, de íntima co-creación, que ninguno puede realmente reclamar como exclusivamente suya 
suyo lo que se haya creado entre ellos. La "Terceridad" se refiere a cómo las mentes de dos sujetos se juegan-de-y-uno con el otroe. Y si pensamos en una "novela, es decir, un libro" como una metáfora para un matrimonio, la cualidad de la "terceridad" en cualquier matrimonio es medido por que tan bien "eso" es "co-escrito". Las estadísticas de divorcio, que exceden el 50\% de los matrimonios en los Estados Unidos, indican cuántas de estas "novelas" han sido "pobremente escritas" y tienen que ser abandonadas (ergo conduciendo al divorcio).

Cuando hablamos de la "terceridad" en psicoterapia, Ogden estipula, con frecuencia la representación del terapeuta se convierte en el "subyugado tercero", en donde ella "subyuga" su mente a aquella del paciente. Ogden nota que esta es la extensión de la naturaleza asimétrica de sus roles. El terapeuta es el "ayudante" y el paciente es el "ayudado". Esto significa que la mayor parte de la atención se dirige a ayudar al paciente.

La posición del tercero subyugado en íntimas relaciones a largo plazo, es decir, matrimonio, pueden ser un poco complicadas. En vez de subyugar la subjetividad de uno por elección, uno puede sentir que debe ceder a la dominación del otro. En otra parte, llamé esto como una especie de "tercero negativo" (Ringstrom, 2001) algo equivalente a la idea de Benjamin de "complementariedad dividida". Esto, por supuesto, es aludido por encima en el segundo tema del reconocimiento mutuo versus negación mutua. La cualidad de la "terceridad" en cualquier relación depende de que tan efectivos sean los dos sujetos para colaborar y negociar desde las posiciones de sus únicas y óptimamente simétricas perspectivas.

A pesar de todo esto, también hay circunstancias en las que el logro de la "terceridad" a veces requiere la capacidad de rendirse. Esto es, porque algunas veces a lo largo de una íntima relación a largo plazo, hay ciertos problemas que simplemente no son negociables. Obviamente, las circunstancias pueden tornarse complicadas en la "división complementaria de dominancia y sumisión de la experiencia de "el hacedor y el hecho para" (Benjamin, 2004, 2018) en la cual paradójicamente ambos compañeros se sienten como el "sumiso", el "hecho para", la "víctima". Es precisamente en estas circunstancias que la capacidad para rendirse puede volverse crítica. Pero esto se vuelve muy complicado, desde que Ghent (1990) argumentó que sumisión y rendición pueden parecerse mucho. Sin embargo, son bastante diferentes, ya que el primero implica una posición masoquista, mientras que el segundo está más informado por un proceso de "dejar ir" algo.

Una manera fácil para distinguir entre sumisión y rendición se encuentra en la distinción entre las preposiciones "para" y "de". En sumisión, sentimos que nos hemos sometido "para" otro, mientras que en rendición sentimos que nos hemos rendido a algo 
"de" nosotros mismos en donde nos hemos sentido atrapados, pero ahora nos sentimos liberados.

Por ejemplo, un marido está fuertemente posicionado en una postura opuesta a la de su mujer acerca de cómo están criando a su hijo adolescente. Ella lo siente a él como dominante y a ella como teniendo que ser sumisa. Sin embargo, después de reflexionar considerablemente, él descubre que su posición es menos sobre algo que él realmente cree, que algún sentimiento que emerge en identificación con cómo su padre lo crio. Esta develación, le permite alcanzar una posición propia, la cual, resulta ser, es más cercana a la de su mujer que aquella que estaba tomando cuando se estaba identificando con su propio padre. Su sumisión lo dirigió a un cambio de su posición "binaria" de "a tu manera o a la mía" a una de "terceridad" involucrando "nuestra manera".

Afortunadamente, es evidente cómo estos tres temas están íntimamente conectados entre sí. Entramos a relaciones esperando que nuestras actualizaciones del self sean reconocidas y respaldadas, mientras que temerlas no lo harán. Así, la calidad de nuestra relación depende en qué tan bien lidiemos con la "paradoja de reconocimiento". Esto es, la paradoja que la pareja tiene que atravesar durante los recurrentes episodios de "negación mutua" mientras están en la búsqueda de reparar sus rupturas en la forma de "reconocimiento mutuo". En esta última, la "terceridad" de la relación con mente propia puede alcanzarse de maneras más saludables y útiles para los compañeros que una vida enfrascada en la "división complementaria" de perpetua "dominancia y sumisión" y quizá la eventual disolución de su relación.

Estos tres temas informan a toda la organización de mi acercamiento psicoanalítico hacia la psicoterapia de pareja. Es un enfoque delineado en Seis Pasos, aunque estos no son "pasos" en el sentido tradicional de una progresión jerárquica lineal. En cambio, deben de pensarse como no lineales, parecido al grabado de Escher (véase al final), en el que uno nota dos líneas disparejas de Monjes que ambos van ascendiendo y descendiendo cuatro escalones, aunque paradójicamente, terminan siempre en el escalón más bajo (es decir, el "primero"). La imagen de no linealidad involucra el genio artístico de Escher, un pie capturado por la ilusión óptica.

Mi concepción de los "seis pasos" es presentado de forma lineal más que nada por motivos organizacionales y menos por motivos prácticos. Los elementos de cualquiera de los Seis Pasos podrían, en varias ocasiones durante el tratamiento, no estar necesariamente en el mismo orden.

Más en el punto, no obstante, los Seis Pasos sugieren un arco del tratamiento que ahora elaboraré. Los pasos del arco progresan hasta que la pareja alcanza cierta maestría expresa. Este material es para uso científico y profesional exclusivamente y puede contener información clínica sensible. Los editores no se responsabilizan de los contenidos de los autores. Dirigir las consultas sobre derechos y autorizaciones a ceir@psicoterapiarelacional.es 
del problema que típicamente había sido el motivo que los llevó a terapia. Mi experiencia, sin embargo, es que una vez que este problema ha sido satisfactoriamente trabajado, nuevos problemas emergían que requerían ciclarse a través de los Seis Pasos nuevamente. Es por ello por lo que los pacientes y terapeutas por igual algunas veces lamentamos, "Después de todo este trabajo, ¡se siente como si hubiésemos vuelto al Paso Uno!" Por supuesto, en cierto sentido es verdad, aunque no es algo por el cual angustiarse. Les aliento a que después de que hayan superado su queja central, que hagan espacio para ellos para analizar los otros problemas. Sin embargo, una vez que han dominado el modelo por medio de la repetición de un problema dispar (aunque conectado), estarán listos para terminar. Hablaré más sobre esto hacia el final de esta presentación, pero primero volvamos a lo que me refería con un arco de tratamiento.

El arco de tratamiento comienza simétricamente (incluyendo los primeros tres pasos) y termina asimétricamente (incluyendo los últimos tres). Esta idea viene de James Grotstein (1997) en donde él asume que toda psicoterapia debería iniciar simétricamente y hasta el final asimétricamente. Con simétrica, Jim quiso decir que nuestros pacientes necesitan experimentar nuestro desarrollo de una poderosa identificación con las principales preocupaciones que los llevaron a terapia. Esto implica involucrarse de una manera profundamente empática, transmitiendo la comprensión del terapeuta lo que las quejas de la pareja significan para cada uno de ellos, en un lenguaje que puedan comprender. Grotstein argumentaba que sólo dentro de estas circunstancias los pacientes pueden sentirse seguros y suficientemente ligados al terapeuta para que verdaderamente divulgue lo que pudieran necesitar. Esto incluye una apertura al surgimiento de material inconsciente, así como a volverse vulnerable a las vicisitudes del cambio que tengan que enfrentar.

Grotstein también decía, sin embargo, que la terapia debe acabar asimétricamente, y para eso él se refería a que debe ir más allá de la simple corroboración de nuestra comprensión del punto de vista del paciente, la primera función de la simetría. Grotstein afirmó que la asimetría crucial implica aseverar, por ejemplo "marcar" (Fonagy, et. al., 2002) la potencialidad y necesidad de diferentes puntos de vista en relación con las quejas de la pareja. Aunque Grotstein no estaba escribiendo sobre terapia de pareja per se, sus aseveraciones son igualmente significativas para el esfuerzo terapéutico de trabajar con cualquier pareja íntimamente comprometida a largo plazo. Siguiendo este arco, postulo, una vez más para enfatizar, que los primeros tres pasos de mi enfoque están en gran parte encarnados en el aspecto simétrico del tratamiento, mientras que los segundos tres pasos expresa. Este material es para uso científico y profesional exclusivamente y puede contener información clínica sensible. Los editores no se responsabilizan de los contenidos de los autores. Dirigir las consultas sobre derechos y autorizaciones a ceir@psicoterapiarelacional.es 
encarnan más el movimiento hacia lo asimétrico. Esto nos lleva a mi delineación de cada uno de los Seis Pasos.

De acuerdo con el objetivo simétrico de formar una fuerte identificación con la experiencia de cada compañero sobre lo que los ha traído a tratamiento, el Paso Uno se centra en la sintonía del terapeuta con la subjetividad de cada miembro de la pareja de forma que busca infundir esperanza, perspectiva y una posibilidad de renovado crecimiento. El Paso Uno está basado principalmente en los principios de la teoría y la práctica derivada de la Psicología del Self y de la Teoría de Sistemas Intersubjetivos. Estas teorías enfatizan que la psicoterapia psicoanalítica debe ser guiada por el compromiso del terapeuta a un método empático/introspectivo. Esto requiere que la terapeuta centre su exquisita sintonía en capturar -en términos cotidianos- lo que Stolorow, et al., refieren como la dimensión selfobjeto reparativa y del desarrollo para la transferencia versus la dimensión repetitiva, temida y resistente de cada uno.

De una manera comparable, Tolpin (2002) se refiere a estas dimensiones bipolares como pertenecientes a un continuo del que ella llama "la experiencia transferencial total". Tolpin describe este continuo como encarnando un "borde delantero" y un "borde trasero" de la experiencia transferencial compuesta. El "borde delantero" es análogo a la transferencia "selfobjeto reparativo y del desarrollo" mientras que el "borde trasero" se acerca a su "dimensión repetitiva y resistente". La transferencia en ambos paradigmas de tratamiento es entendida como implicando la organización fundamental de todo individuo del sentido del self en relación con su sentido del otro, así como lo que ellos imaginan que el otro siente sobre ellos. Estas ideas sobre la transferencia no son tan simples como parecen. Esto es porque el sistema de transferencias también incluye la del terapeuta, comúnmente referida como las dos dimensiones de la contratransferencia del terapeuta.

Y como si el asunto no fuera más complicado, el psicoanálisis relacional también ve nuestra personalidad como compuesta por múltiples estados del self. Como lo pone Philip Bromberg (1998), "nos sentimos como uno, mientras somos muchos". Esto se vuelve una idea muy esencial en el lado asimétrico del arco, aunque con frecuencia aludo pronto a la idea de múltiples estados del self en el tratamiento.

Sin embargo, es en la capacidad del terapeuta el identificar los respectivos "bordes delanteros y traseros" de la queja de cada compañero, que el tema de sus respectivas "esperanzas y temores" sea revelada. De esta manera, el terapeuta no solo desarrolla una poderosa identificación cada una de sus experiencias de "esperanza y temor", incluso más importante, también demuestra con fuerza qué es lo que está pasando para que se estén activando sus estados de transferencia. Para hacer esto, ella señala como el borde 
"delantero" (los deseos de esperanza) de la transferencia de cada compañero detona los bordes "traseros" (la experiencia de temor/miedo) el uno del otro, vinculando rápidamente la experiencia de "esperanza" con una de "temor" de un modo circularmente reforzador.

Los Pasos Dos y Tres, aumentan lo que se lleva a cabo en el Paso Uno. El Paso Dos está profundamente anclado en la idea epistemológica del "realismo perspectivista"f en el que debemos acceder a la idea de que en realidad hay algo a lo que todos nos referimos y que hemos llamado "realidad". Esto es algo en lo que estamos de acuerdo en que verdaderamente existe, a pesar del hecho de que nuestra experiencia subjetiva de "eso" puede diferir considerablemente. Aunque simultáneamente, debemos reconocer que lo que sea que sea "eso" a lo que nos referimos como "realidad", es "algo" que nadie puede aprehender en su totalidad. A lo mucho, cada uno de nosotros podemos tener nuestra perspectiva única de una "porción de ella". Por lo tanto, las diferencias en nuestras perspectivas son abundantes $y$, sin embargo, completamente esperables.

Esto entonces, es el roce esencial de la naturaleza humana y lo relacional. En diferentes grados, estamos de acuerdo o en desacuerdo con nuestras respectivas perspectivas -esa a la que colectivamente llamamos "realidad". El consenso de desacuerdos en el tratamiento de parejas pone de relieve por qué hablo de ello como siempre involucrando una "guerra de epistemologías". En casos más extremos, en donde las diferencias de puntos de vista acerca de lo que es "verdad" y "real" son de gran importancia, los compañeros pueden terminar sintiendo que su cordura está siendo cuestionada. Cada compañero puede encontrarse a sí mismo en un conflicto que Jody Davies (2003) describió como cuando cada uno siente que: "para sentirme cuerdo debo olvidar el amor y para sentirme amado, debo olvidar mi cordura".

El problema del perspectivismo no está aislado únicamente a la perspectiva de los compañeros, pero incluye el grado en el que la perspectiva del terapeuta culmina en su contratransferencia y en las "conjunciones y disyunciones" con la perspectiva de sus pacientes. La contratransferencia conjuntiva implica que el punto de vista subjetivo del terapeuta sea correspondiente con aquella de la pareja que está obstruyendo su capacidad para reconocer, y mucho menos, afirmar la diferencia significativa. Para evocar un elemento idiomático inglés, ella se vuelve incapaz de "ver el bosque por los árboles". En cambio, la contratransferencia disyuntiva implica que haya cierto grado de diferencia entre la perspectiva del terapeuta y la de la pareja, que sean incapaces de encontrar un terreno en común.

En la terapia de pareja es normal que la contratransferencia conjuntiva y disyuntiva se alcen. Lo que es crítico, sin embargo, es para el terapeuta de aceptarlo cuando ocurre. El expresa. Este material es para uso científico y profesional exclusivamente y puede contener información clínica sensible. Los editores no se responsabilizan de los contenidos de los autores. Dirigir las consultas sobre derechos y autorizaciones a ceir@psicoterapiarelacional.es 
no hacerlo podría llevar al compañero en la posición disyuntiva a amenazar con terminar la terapia o entregarse a volverse patológicamente acomodado después de comprometerse en una especie de pseudo-tratamiento que lleve a la terapia a un impasse. Sin embargo, cuando el terapeuta reconoce abiertamente sus sesgos, ella comienza a demostrar como semejante controversia puede retomarse de varias maneras constructivas, que aún no son parte del repertorio de la pareja.

La esencia del Paso Dos, entonces, es el cultivo de una atmósfera más comprometida a la curiosidad, particularmente con respecto a la iluminación de, colaboración de y negociación de las diferentes perspectivas de los integrantes de la pareja. Una parte clave del arco simétrico del enfoque de tratamiento es la delegación de la tendencia de los compañeros a convertirse en la "complementariedad dividida" de "dominio versus sumisión" aludida en el Segundo Tema del enfoque.

El material de fondo acumulado en el Paso Tres aumenta el aspecto orientado a la sintonización consciente del proceso de simetría, mientras es reforzado por una serie de ideas del psicoanálisis y otras ciencias sociales que nos ayudan a comprender mejor los elementos clave de las aportaciones de cada compañero en lo que respecta a una comprensión enriquecida de la perspectiva subjetiva de cada compañero. Los datos obtenidos del Paso Tres del desarrollo de cada compañero puede ocurrir a través de la interacción in vivo en el "aquí y ahora" del tratamiento, o bien, sesiones deliberadas de toma de antecedentes como se describe en mi libro.

Las ideas clave acerca de los antecedentes de cada compañero incluyendo su capacidad de "mentalización", su exhibición de varios grados de patrones de apego de "seguridad e inseguridad", su uso de los sistemas de memoria "implícita versus explícita", así como a la influencia cultural y de género. Un ejemplo de las influencias de la cultura, podrían implicar el grado el cual los compañeros pertenecen a culturas "colectivistas" versus "individualistas". Todas estas ideas se recogen a detalle in el Paso Tres de mi libro.

Es con el avenimiento de la segunda mitad del arco, cuando el tratamiento cambia de simétrico a asimétrico, que podemos ver la introducción del "giro relacional" en el Paso Cuatro hasta el Seis. Si bien la profundidad de la sintonización empática permite la poderosa identificación del terapeuta con cada compañero, hasta cierto nivel, el tratamiento podría verse limitado por las "historias" conscientes de la pareja sobre lo que creen que implican sus problemas.

Antes de ahondar en el "giro relacional" debe tenerse en cuenta que se puede lograr un trabajo considerable en la simetría de los Pasos Uno a Tres, y que a veces las parejas consideran que este trabajo es suficiente. Están satisfechos de haber desarrollado un 
sentido más profundo de su propia subjetividad, así como la de su pareja. Y, como resultado, descubren que están mejor equipados para colaborary negociar su relación sobre esta base. Esto se vuelve suficiente para ellos para terminar.

Mientras que la mitad simétrica del arco de tratamiento es necesario, para la mayoría de las parejas es insuficiente. Esto es porque, la fase simétrica deja en el "piso de sala de edición" material intrapsíquico disociado. Desde el punto ventajoso del psicoanálisis relacional, esto incluye el material inconsciente de los disociados múltiples estados del self que típicamente se revelan en la forma de enactments. Con frecuencia, estos enactments pueden ponerse en juego después de que la parte simétrica del tratamiento haya hecho que el trabajo sea lo suficientemente seguro y conectado para que se trate el material inconsciente más perturbador. Para complicar aún más este proceso, el terapeuta se ve atrapado en los enactments, algunas veces a través de sus propias disociaciones.

Desde la perspectiva relacional, sin embargo, los enactments no son vistos como "pasos en falso", a lo mucho como presentando el tratamiento con momentos oportunos. En el último caso, estos desvelan "pensamientos no pensados" (Bollas, 1989) y "experiencias no formuladas" (Stern, 1997). Los estados disociados del self pueden emerger en la experiencia de "estados no-yo". Esto puede implicar experimentar algo que no se siente como "de mi" pero, claramente, alguna parte de mí siente que "eso" es. Es a través de la iluminación de estos estados que su lugar en la totalidad de la personalidad de cada compañero comienza a tener sentido. Como Bromberg entona, esto mueve el proceso terapéutico de la disociación de cada compañero a la realización de sus respectivos conflictos intra-subjetivos.

Todo esto es lo que hace que el Paso Cuatro aparezca como el "despertar de los gigantes durmientes" dentro del proceso de tratamiento. A comparación del lado simétrico del arco que trata sobre "el temor de repetir la transferencia negativa" de los pacientes, el "giro relacional" asimétrico implica el "temor a no repetirlo". La última necesidad se relaciona con el siguiente axioma: "Que las parejas no pueden auténticamente negociar entre sí (intersubjetivamente), lo que no han negociado dentro de sí mismos (intrasubjetivamente)".

Esto nos lleva al Paso Cinco que recoge el reconocimiento y la negociación de conflictos entre los múltiples estados del self de cada compañero que no se han tenido en cuenta. Podríamos referirnos a esto como la iluminación intrapsíquica o "intrasubjetiva" del modelo de tratamiento. Este paso implica el enriquecimiento de la capacidad de actualización del self de cada compañero a través de una introspección mejorada en sus conflictos. Y, lo que es especialmente convincente, es que lo hacen en la presencia real del 
otro. Este trabajo implica transformar "estados disociados del self" en "estados distribuidos" (Pizer, 1998) o lo que he denominado como el trabajo de iluminar múltiples estados del self que evolucionan en algo así como un "comité mental". Un espacio análogo en el que finalmente se reconocen los conflictos entre los estados del self, haciendo espacio para sus posiciones de colaboración y negociación representativas de algo que se asemeja a una "voz" compuesta de los múltiples estados del self.

Es a través del creciente sentido de cada compañero de la multiplicidad de su personalidad, así como al enfrentar ciertos aspectos conflictivos de su sentido del self, que se fortalecen para buscar compromisos más auténticos el uno con el otro. Además, a través de esto, están mucho mejor equipados para colaborar y negociar, incluyendo el cálculo de aspectos de sí mismos que no pueden negociarse $y$, por lo tanto, pueden requerir asumir un lugar de "rendición" como se detalla en el tercer tema, la relación teniendo una mente propia.

A medida que se avanza en el trabajo de los aspectos conflictivos de la mente intrasubjetiva de cada compañero, comenzamos a ver la capacidad de cada uno para sintonizar con y apoyarse mutuamente en su introspección y crecimiento personal. Este movimiento en el Paso Cinco, es el precursor para el Paso Seis, el establecimiento del escenario para una mayor colaboración intersubjetiva y la negociación de sus conflictos. Finalmente, en el caso de ciertas diferencias irreconciliables, aumenta su capacidad para conciliar y facilitar un sentido de rendición personal, sin caer en la complementariedad de la sumisión a sentirse dominado.

En el Paso Seis, el concepto del terapeuta como representante de la "terceridad" el crucial punto de vista del "tercero" a lo largo del tratamiento - se cristaliza por completo. En el Paso Seis, vemos que ha sido en el papel de la "terceridad" del terapeuta que ella ha sido especialmente efectiva en facilitar la resolución de una variedad de binarios que hasta ahora habrían fijado a la pareja en la complementariedad de la "dominancia y sumisión". Es decir, su historial de argumentos que se han transformado en ninguno/o y no ambos/y.

Estos últimos tienden a degenerar en irreconciliables argumentos "blanco y negro" sobre cuestiones como la crianza de los hijos, el sexo, las finanzas, los compromisos con sus respectivas familias de origen, el sadomasoquismo, etc. El concepto de "terceridad" crea una perspectiva en la que ambos compañeros se dan cuenta de que se sienten como la "víctima" del otro, lo que les impone una especie de paradoja. La única manera en la que ambos pueden sentirse como "víctimas" es reconociendo también que ambos están siendo sentidos por el otro como "persecutores". La terceridad emerge cuando ambos reconocen qué es lo que se siente sobre ellos como "persecutorio". Es decir, lo que hace que el otro se 
sienta "victimizado". A través de este reconocimiento, comienzan a ver que son "lo mismo". Que sus posiciones frente a frente el uno del otro encarnan tanto al "persecutor" como a la "víctima", que se gestan en algún nivel de reconocimiento mutuo. Esto les permite ir más allá de los patrones fijos de tratarse como "objetos" para tratarse como "sujetos". Aquellos que se sienten reconocidos independientemente de lo que aún pueda persistir de sus diferentes perspectivas9. O, como dice Jessica Benjamin, se mueven "más allá del "hacedor" y del "hecho para" (Benjamin, 2004, 2018).

La conclusión del Paso Seis también sirve como una forma de pensar acerca de la terminación, que implica la transferencia exitosa de la función terapéutica del modelo de Seis Pasos a la pareja. Esto ocurre cuando la pareja ha internalizado lo suficiente los pasos como para utilizarlos por sí solos. Ya no necesitan idealizar, y mucho menos necesitar, aferrarse al terapeuta como el "tercero". Ahora son capaces de mantener la "terceridad" por sí mismos.

En todo esto, el Paso Seis presagia la idea de la terminación. Para cuando la pareja haya pasado por el proceso de los Seis Pasos unas pocas veces, comenzaran a adueñarse de los pasos a tal punto que el terapeuta y la terapia dejan de ser necesarias. En el lugar del terapeuta hay una "entidad estructural" experiencial a la que pueden referirse cuando se encuentran, una vez más, yendo por el horrendo camino de estar atrapados en el mundo binario de "blanco y negro", "lo correcto y lo incorrecto", "el bien y el mal".

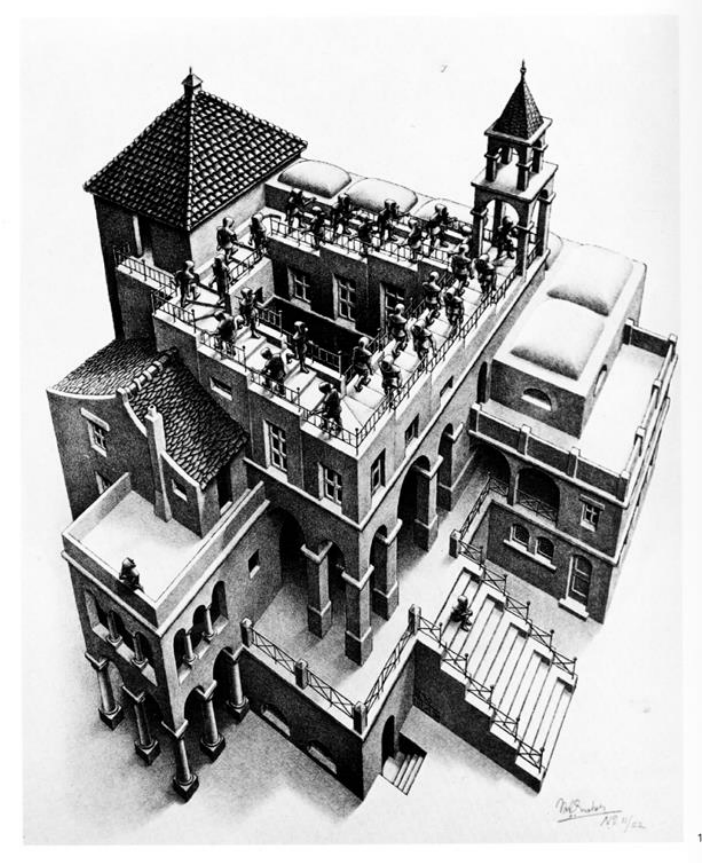
expresa. Este material es para uso científico y profesional exclusivamente y puede contener información clínica sensible. Los editores no se responsabilizan de los contenidos de los autores. Dirigir las consultas sobre derechos y autorizaciones a ceir@psicoterapiarelacional.es 


\section{REFERENCIAS}

Benjamin, J. (1988). The Bonds of Love. New York: Pantheon Books.

Benjamin, J. (2004). Beyond Doer and Done to: Recognition and the Intersubjective Third. Psychoanal. Quar.

Benjamin, J. (2018). Beyond Doer and Done to: Recognition Theory. Intersubjectivity and the Third. London: Routledge.

Bollas, C. (1989). The Forces of Destiny. Northvale, NJ: Jason Aronson Inc.

Bromberg, P. (1998). Standing in Spaces: Essays on Dissociation, Trauma and Clinical Process, Hillsdale NJ: The Analytic Press.

Davies, J. (2003). Falling in love with love: Oedipal and postoedipal manifestations of idealization, mourning and erotic masochism. Psychoanalytic Dialogues. 13, 1-27.

Fonagy, P., Gergeley, G., Jurist, E. and Target, M. (2002). Affect regulation, mentalization, and the development of the self. New York: Other Press.

Ghent, M. (1990). Masochism, Submission and Surrender. Contemporary Psychoanalysis. Vol. 26, No. 1. 108-135. [versión castellana en CeIR]

Gleick, J. (1987). Chaos: Making A New Science. New York: Viking Penguin Press.

Goldner, V. (2014). Romantic Bonds, Binds, and Ruptures: Couples on the Brink. Psychoanalytic Dialogues. New York: Taylor and Francis Group. 24: 402-418.

Grotstein, J. (1997). Autochthony and alterity: psychic reality in counterpoint. Psychoanalytic Quarterly, LXVI, pp. 403-430.

Lichtenberg, J. D., Lachmann, F. L., and Fosshage, J. L., (1992). Self and Motivational Systems: Towards a Theory of Psychoanalytic Technique. Vol. 13, Hillsdale, NJ: The Analytic Press.

Lichtenberg, J. D., Lachmann, F. L., and Fosshage, J. L., (1996). The Clinical Exchange: Techniques Derived from Self and Motivational Systems. Hillsdale, NJ: The Analytic Press.

Mitchell, S. (1993). Hope and Dread in Psychoanalysis, New York: Basic Books.

Mitchell, S. (2000). Relationality, Hillsdale, NJ: The Analytic Press.

Ogden, T. (1994). Subjects of Analysis. Northvale NJ: Jason Aronson, Inc. Prol

Pizer, S. (1998). Building Bridges: The Negotiation of Paradox in Psychoanalysis. Hillsdale, NJ: The Analytic Press.

Ringstrom, P. (1994). An intersubjective approach to conjoint therapy. In: Progress in Self Psychology, Vol. 10. Ed. A. Goldberg, Hillsdale, NY: The Analytic Press.

Ringstrom, P. (2001). The Noxious Third: The Crimes and Misdemeanors in the Treatment of Tony Soprano and Dr. Jennifer Melfi. $21^{\text {st }}$ Annual Spring Meeting of Division of Psychoanalysis [39]. Santa Fe, NM.

Ringstrom, P. (2012). A Relational Intersubjective Approach to Conjoint Therapy. Intl J. of Psychoanal. Self Psych. 7: 85-111. 
Ringstrom, P. (2014). A Relational Psychoanalytic Approach to Couples Psychotherapy. London: Routledge.

Stern, D. B. (1997). Unformulated Experience: From Dissociation to Imagination in Psychoanalysis. Hillsdale, NJ: The Analytic Press.

Stern, D.B. (2015). Relational Freedom: Emergent Properties of the Interpersonal Field. New York and London: Routledge.

Tolpin, M. (2002). Chapter 11. Doing Psychoanalysis of Normal Development: Forward Edge Transferences. Progress in Self Psychology, 18:167-190.

Original recibido con fecha: 2/10/2017 Revisado: 21/1/2018 Aceptado: 28/02/2018

NOTAS:

a N. del T. En Estados Unidos de América hay dos tipos de programa doctoral en psicología. El PH.D. es un doctorado de Filosofía en Psicología e involucra más el plano académico y de investigación, mientras que el PSY.D. es un doctorado en psicología que va destinado a aquellos que se han enfocado a la práctica clínica.

${ }^{\text {b } L o ~ q u e ~ o f r e c e ~ e l ~ p s i c o a n a ́ l i s i s ~ r e l a c i o n a l ~ e s ~ u n ~ t i p o ~ d e ~ m e t a-t e o r i ́ a, ~ u n a ~ v e r d a d e r a ~ " c a r p a ~ g r a n d e " ~ e n ~ l a ~ q u e ~ c a d a ~}$ teoría contemporánea del psicoanálisis puede cobijarse, como, por ejemplo, las versiones contemporáneas del freudiano, kleiniano, relaciones objetales, psicología del self, interpersonal, lacaniano, junguiano, etc., retoma las implicaciones de lo que significa que todas las metapsicologías anteriores se hayan convertido en teorías psicológicas prácticas de "múltiples personas" -aunque en su propia manera particular.

'Ringstrom, P. (en proceso) Three-Dimensional Field Theory: Dramatization and Improvisation in a Psychoanalytic Theory of Change. (entregado a Routledge Publishing).

${ }^{d}$ Gleick (1987) apunta en su libro Chaos: Making A New Science que los meteorólogos, que estuvieron entre los primeros en hacer uso de la teoría del caos, también conocida como teoría de la complejidad, postulaba que, teóricamente, los orígenes de un huracán en El Caribe podrían haber iniciado por el batir de alas de una mariposa en Venezuela.

e Sobre este punto, Goldner (2014) escribe: "En efecto, si has estado viviendo y durmiendo con tu pareja por dos años (no debería sorprendernos que sólo nos apeguemos a los que tocamos), ahora estás unido, rodeado de el uno al otro, de sistema nervioso a sistema nervioso, y su estado psíquico ahora es propiedad conjunta. Puede que no sea feliz, puede que no sea bueno, pero a pesar de nosotros mismos, es nuestra naturaleza humana, parafraseando a Crosby, Stills, Nash \& Young: el "amo a aquel con quien estoy".

f Esta perspectiva, a veces se denomina como "constructivismo social" o "construccionismo de perspectiva" (aunque los tres difieren un poco). Sin embargo, todos ellos comparten un punto de vista epistemológico que encarna una especie de paradoja.

9 Esta "terceridad" adopta diversas formas diferentes, tal como Benjamin (2004) escribió de forma destacada, el "uno-en-el-tercero" perteneciente a la "ritmicidad", como en "hacen falta dos para el tango." Aquí, el tango representa una poderosa metáfora mediante la cual la pareja aprende finalmente como "bailar" juntos en la medida en que el papel de cada uno se basa firmemente en seguir las pistas y las respuestas de los otros de una manera que crea la estética de su nueva danza. Esto contrasta con lo que Benjamin describió como el "tercero en uno" que implica los procesos que marcan las diferencias subjetivas de los miembros de la pareja, en el contexto de su creciente conexión. 